\title{
A Performance Evaluation Framework of A Rate-Controlled MPEG Video Transmission over UMTS Networks
}

\author{
N. Akar ${ }^{(1)}$, M. Barbera $^{(2)}$, L. Budzisz ${ }^{(3)}$, R. Ferrùs ${ }^{(3)}$, E. Kankaya ${ }^{(1)}$, G. Schembra ${ }^{(2)}$ \\ (1) Bilkent University, (2) University of Catania, $\quad{ }^{(3)}$ Universitat Politècnica de Catalunya \\ e-mail: akar@ee.bilkent.edu.tr, mbarbera@diit.unict.it, lukasz@tsc.upc.edu, \\ ferrus@tsc.upc.edu,emre@ee.bilkent.edu.tr, schembra@diit.unict.it
}

\begin{abstract}
UMTS is designed to offer high bandwidth radio access with QoS assurances for multimedia communications. In particular, real-time video communications services are expected to become a successful experience under UMTS networks. In this context, a video transmission service can be designed over the basis that UMTS can provide either a constant bit rate data channel or a dynamic variable bit rate data channel adapted to load conditions. In this latter approach, which is more efficient for both the user and the service provider, multimedia sources have to be timely designed in order to adapt their output rate to the instantaneous allowed channel rate. The target of this paper is to define an analytical model of adaptive real-time video sources in a UMTS network where system resources are dynamically shared among active users.
\end{abstract}

KEYWORDS: Video, UMTS, Markov Models, Performance Evaluation, QoS

\section{INTRODUCTION}

Universal Mobile Telecommunication System (UMTS) has been defined to provide the third generation of mobile telecommunication systems. UMTS has its out-spring from the second generation system GSM. It is designed to offer high bandwidth radio access for multimedia communications as well as the traditional voice services, and to provide a wide range of bearer services with different levels of Quality of Service suitable for multimedia applications with bit rates ranging from $64 \mathrm{~kb} / \mathrm{s}-2 \mathrm{Mb} / \mathrm{s}$ in different environment conditions. In the UMTS radio interface, data generated at higher layers is carried over the air with transport channels, which are mapped in the physical layer to different physical channels. The physical layer is required to support variable bit rate transport channels to offer bandwidth-on-demand services, and to be able to multiplex several services to one connection. This flexibility is achieved within the physical layer by supporting different transport block sizes, CRC code lengths, channel coding schemes, transport time intervals, rate matching and spreading factors. The perceived quality of the application seen by the end user is greatly affected by the settings of these radio access parameters.

An important issue to be accounted is that the chan-

The authors would like to acknowledge the funding from EU FP6 IST-NEWCOM project. nel bandwidth seen by each user is time-variant, and therefore traffic sources have to adapt their output rate to follow the channel behavior. To this aim, each source has to determine channel distortion before transmitting each piece of information, and adapt encoder/network parameters in a way that maximizes the quality of the received video at the decoder.

In this context, designing multimedia sources in order to be able to adapt their output rate to the strongly variable channel conditions optimizing quality perceived at destination, will be challenging for future research. To this aim, a central role is played by Rate Controller which works tightly coupled with the encoder to appropriately set its encoding parameters [1][2]. Rate Controller monitors the available bandwidth, the activity of the frame which is being encoded, the encoding mode, and the state of the transmission buffer to take into account the amount of data used to encode the previous frames; then it chooses the appropriate parameters in such a way that the transmission buffer at the source never saturates. The relationship between the encoding parameters and the above information is the socalled feedback law.

The target of this paper is to define an analytical model of real-time video sources over UMTS Terrestrial Radio Access Network (UTRAN), with the aim of studying the effects of the conditions of the UMTS uplink channel on the performance of realtime video communications. To this aim, the whole system is modeled as an emission process feeding the transmission buffer; the server of this buffer behaves according to the channel conditions, and in particular, the available bandwidth. Particularly, the service rate is directly proportional to the available channel bandwidth per user. We denote the whole transmission system model as $\mathrm{SBBP} / \mathrm{SBBP} / 1 / K$ since it is a queuing system model characterized by two switched batch Bernoulli processes (SBBP) [3], one modeling the traffic at the input of the buffer, and the other modeling the buffer queue server; $K$ represents the buffer queue dimension. In our case, the queuing system is rate controlled since the first SBBP behavior is controlled by the state of the queue. The analytical model can be applied to the design of both the source encoding parameters, such as the feedback law, and some network parameters, 
such as the maximum number of users in the UMTS cell.

The paper is structured as follows. Section 2 describes the considered system, which is constituted by a UMTS network populated by mobile video users. Section 3 presents the proposed model. The model is then applied to a case study, and Section 4 provides some numerical results. Finally, Section 5 concludes the paper.

\section{SYSTEM DESCRIPTION}

In this section we model a UMTS cell loaded by rate-controlled MPEG video (RCMV) sources, as shown in Fig. 1. Assuming that all sources present the same statistical characteristics, in order to capture the overall behavior and to evaluate performance of both each source and the system as a whole, we focus our attention to a given video source, in the following referred to as tagged source (TS). As shown in Fig. 1, it is an MPEG video source whose MPEG encoder is controlled by a rate controller. The target of the rate controller is to choose the quantizer scale parameter (QSP), $q$, to be used to encode each frame, starting from the knowledge of the activity value $a$ of the frame which is being encoded, its encoding mode $j$, and the number of packets $s_{Q}$ which are present in the transmission buffer. This is obtained by implementing the feedback law $q=\phi\left(s_{Q}, a, j\right)$. The definition of the feedback law depends on the target we want to pursue. For example, we can address the target of achieving the same number of packets for each encoded frame, on average, in order to minimize the burstiness of the traffic sent over the network. Another feedback law can be defined with the target of obtaining a constant number of packets per Group of Pictures ( $\mathrm{GoP}$ ) leaving the number of packets for encoding each frame in the GoP variable in order to pursue a constant distortion level within the GoP. The output flow of the MPEG encoder is then packetized and sent to the Transmission Buffer. The emission process of the transmission buffer server depends on the channel transmission capacity and, in particular, coincides with the time-varying available bandwidth, as will be discussed later.

\section{SYSTEM MODEL}

To model the system described in Section 2 we first use a discrete-time approach, where the frame duration, $\Delta$, is used as the time slot. This discrete-time approach will then be replaced with a fluid-flow approach for numerical analysis. Let us indicate the number of active video users in the UMTS cell at the generic slot $n$ as $U(n)$, and assume that $U(n)$ varies according to a finite birth-death discrete-time Markov model, whose behavior is described by the rates of a birth, $r_{b}$, and a death, $r_{d}$. Let $u_{M A X}$ be the

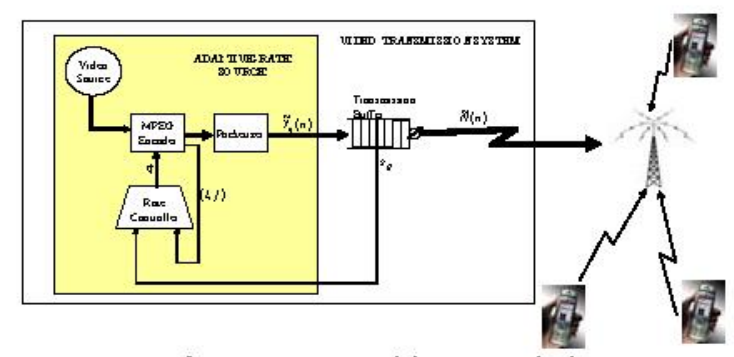

Figure 1. Reference UMTS Video transmission system.

maximum number of users in the cell, and $Q^{(U)}$ the transition probability matrix of the process $U(n)$. The adaptive-rate MPEG video source is modeled as an $S B B P / S B B P / 1 / K$ queuing system, where the first SBBP, $\widetilde{Y}(n)$ represents the arrival process to the transmission buffer, the second SBBP, $\tilde{N}(n)$, represents the service process, and $K$ is the maximum number of packets the video source transmission buffer can contain.

Section 3.1 briefly defines the SBBP model and some notation. Then we introduce the models of the non-controlled video source (Section 3.2), the UMTS channel (Section 3.3), and finally the overall system (Section 3.4).

\subsection{Switched batch Bernoulli process (SBBP)}

An SBBP $Y(n)$ is a discrete-time emission process modulated by an underlying Markov chain. Each state of the Markov chain is characterized by an emission probability density function (pdf): the SBBP emits data units according to the pdf of the current state of the underlying Markov chain. Therefore an $\operatorname{SBBP} Y(n)$ is fully described by the state space $\Im^{(Y)}$ of the underlying Markov chain, the maximum number of data units the SBBP can emit in one slot, $r_{M A K}^{(Y)}$, and the set $\left(Q^{(Y)}, B^{(Y)}\right)$, where $Q^{(Y)}$ is the transition probability matrix of the underly ing Markov chain, while $B^{(Y)}$ is the emission probability matrix whose rows contain the emission pdf's for each state of the underlying Markov chain. Below we will introduce an extension of the meaning of the SBBP to model not only an emission process, but also the activity process which characterizes the video sequence. In the latter case, we will indicate it as an activity $S B B P$, and its matrix $B^{(Y)}$ as the activity probability matrix.

\subsection{Non-controlled video source model}

Let us define the SBBP process $\tilde{Y}_{q}(n)$, modeling the emission process of the non-controlled MPEG video source at the packetizer output for each quantizer scale parameter (QSP) $q \in[1,31]$. This model was proposed in [3][4]. So, for the sake of completeness, 
here we will only define notation.

The model captures two different components: the activity process behavior and the activity/emission relationships. As input it takes the first- and secondorder statistics of the activity process, and the three functions, one for each encoding mode ( $\mathrm{I}, \mathrm{P}$ or $\mathrm{B}$ ), characterizing the activity/emission relationships. The state of the underlying Markov process of $\widetilde{Y}_{q}(n)$ is a double variable, $S^{(\tilde{Y})}(n)=\left(S^{(G)}(n), S^{(F)}(n)\right)$, where $S^{(G)}(n) \in \mathfrak{J}^{(G)}$ is the state of the underlying Markov chain of the activity process $G(n)$, and $S^{(F)}(n) \in J$ is the frame to be encoded in the GoP at the slot $n$. The state set $\mathfrak{\Im}^{(G)}$ represents the set of activity levels to be captured. The set $J$, on the other hand, represents the set of frames in the GoP and depends on the GoP structure assumed. As demonstrated in [3], the underlying Markov chain of $\widetilde{Y}_{q}(n)$ is independent of $q$. Therefore, we will indicate its transition probability matrix as $Q^{(\widetilde{Y})}$ instead of $Q^{\left(\tilde{Y}_{q}\right)}$, and the set $\left(Q^{(\widetilde{Y})}, B^{\left(\tilde{Y}_{q}\right)}\right)$, for each $q \in[1,31]$, completely defines the SBBP emission process modeling the output flow of the noncontrolled MPEG encoder, when it uses a fixed QSP, $q$.

\subsection{Channel model}

The target of this section is to define the SBBP process modeling the channel capacity available to the TS source. The smallest entity of traffic that can be transmitted through a transport channel is a Transport Block (TB). In a certain period of time, called a Transmission Time Interval (TTI), a given number of TBs will be delivered to the physical layer, which depends on some coding characteristics, interleaving, and rate matching to the radio frame. It determines the effective bit rate $R_{b}^{i}$ (bits/s) seen by a video source $i$ at the application level. The maximum allowed bit rate depends mainly on the number of active users (i.e. interference level), the bit energy over noise ratio $\left(E_{b} / N_{0}\right)$ to be guaranteed, and the propagation loss between the mobile and the BS. In the following we will assume that mobile terminals are not power limited, in such a way that they are always able to compensate their path loss by transmitting the needed power. Therefore we will assume that maximum allowed bit rate only depends on the number of users which are present in the cell, and the bit energy over noise ratio, $E_{b} / N_{0}$, to be guaranteed.

According to the user transmission policy described later on in this section, it is claimed that the bit rate seen by the TS source can be described by an SBBP model, modulated by the process $U(n)$ of the number of video users active in the cell. We will consider the TB as the SBBP emission unit. Let $\left(Q^{(\tilde{N})}, B^{(\tilde{N})}\right)$ be its parameter set. The transition probability matrix is $Q^{(\widetilde{N})}=Q^{(U)}$. The matrix $B^{(\widetilde{N})}$ can be calculated by the probabilities that $R_{b}^{i}$ bits/second, or its equivalent number of TBs, are transmitted in one slot, for each state of $U(n)$. Let us now calculate these probabilities. It can be stated that radio resources in a UMTS uplink channel are assigned to user $i$ in terms of data rate $\left(R_{b}^{i}\right)$ and transmission power $\left(P_{T}^{i}\right)$, and this assignment might vary on a TTI timescale basis (10 ms or multiples of it) [5]. In the following we assume that all the users have the same requirements on the maximum admissible BLER (Block Error Ratio). This requirement determines the minimum value of the bit energy over noise ratio $\left(E_{b} / N_{0}\right)_{i}^{M I N}$ with a relationship depending on propagation channel conditions, mobile's speed and diversity techniques. In CDMA systems, the bit energy over noise ratio for a given user $i$ is obtained as:

$$
\left(\frac{E_{b}}{N_{0}}\right)_{i}=\frac{W}{R_{b}^{i}} \frac{P_{R}^{i}}{I_{\text {Total }}-P_{R}^{i}}
$$

where $W$ is the system bandwidth, $I_{\text {Total }}$ is the total received wideband power including thermal noise power at the BS receiver, and $P_{R}^{i}$ is the power received at the BS from the user $i$. Thus, considering that $\left(E_{b} / N_{0}\right)_{i}$ has to satisfy the constraint $\left(E_{b} / N_{0}\right)_{i} \geq\left(E_{b} / N_{0}\right)_{i}^{M N}$, for each user $i$, the minimum value required for $P_{R}^{i}$ can be derived from (1) as:

$$
P_{R}^{i} \geq \frac{1}{1+\frac{W}{\left(E_{b} / N_{0}\right)_{i}^{M I N} R_{b}^{i}}} I_{\text {Total }}
$$

Power control in uplink will be in charge of adjusting mobile transmission power for (2) to be satisfied. More specifically, assuming perfect power control, the power transmitted by user $i, P_{T}^{i}$, can be calculated as $P_{T}^{i}=\min \left(P_{R}^{i} \cdot L_{p}^{i}, P_{T \text { max }}^{i}\right)$, where $L_{p}^{i}$ is the propagation loss between mobile $i$ and the BS, and $P_{T \max }^{i}$ is the maximum allowed transmission power. If in the generic slot $n, U(n)=\bar{u}$ users are connected to an UMTS base station, the following expression must be held for the total amount of power received from mobile users if inter-cell interference is not accounted:

$$
\sum_{i=1}^{\bar{u}} P_{R}^{i}=\sum_{i=1}^{\bar{u}} \frac{1}{1+\frac{W}{\left(E_{b} / N_{0}\right)_{i}^{M N N} R_{b}^{i}}} I_{\text {Total }} \leq I_{\text {Total }}
$$

From (3) we then obtain the condition to be satisfied in terms of number of users in such a way that 
each user in the cell can obtain the expected quality:

$$
\sum_{i=1}^{\bar{u}} \frac{1}{1+\frac{W}{\left(E_{b} / N_{0}\right)_{i}^{M N N} \cdot R_{b}^{i}}} \equiv \eta_{\text {UL }}<1
$$

where $\eta_{U L}$ is the uplink load factor that captures the amount of supported traffic in the UMTS channel. When $\eta_{\text {UL }}$ becomes close to 1 , the system has reached its pole capacity. Usually this load factor is used within admission and load control in the system to avoid system instabilities, by not allowing $\eta_{U L}$ to exceed a given threshold $\eta_{U L}^{T h}$ (e.g. keeping $\eta_{U L}$ below $\eta_{U L}^{T h}=0.7$ makes the system to operate below the $70 \%$ of the pole capacity). So, if no power limitations are considered in mobile terminals and power control behaves ideally, expression (4) can be used as the channel capacity bound to be satisfied for a given $\eta_{U L}^{T h}$ target. Notice that if this bound is exceeded, some of the terminals in the system would not achieve the minimum required $\left(E_{b} / N_{0}\right)_{i}^{M I N}$. So, once the overall channel capacity is fixed, now the problem is how this capacity is shared among users. Two important assumptions are done here: (1) during a video session, the transmission buffer of the mobile never gets empty because the rate controller continuously fills it; (2) mobile terminals would try to transmit so that the buffer is emptied as soon as possible. These assumptions lead to the fact that mobile terminals always try to transmit at maximum available channel capacity. Then, if deployed radio resource allocation strategies (i.e. scheduling) are able to assure fairness, the overall channel capacity can be assumed to be equally distributed among all the users. Thus, for $U(n)=\bar{u}$ users with identical $\left(E_{b} / N_{0}\right)_{i}^{M N}$ requirements (i.e equal to $\left(E_{b} / N_{0}\right)^{M N}$ for all the users), the allowed channel rate per user is calculated from (4) as:

$$
R_{[\bar{u}]}^{(b)}=\frac{W}{\left(E_{b} / N_{0}\right)^{M N}} \cdot \frac{\eta_{U L}^{T h}}{\bar{u}-\eta_{U L}^{T h}} \quad \forall \bar{u} \in\left[0, u_{M A X}\right]
$$

Finally, the emission probability matrix of the SBBP channel model can be calculated as follows. Let $H$ be the transmission packet size, and therefore $R_{[\bar{u}]}^{(P)}=R_{[\bar{u}]}^{(b)} \cdot \Delta / H$ the channel capacity expressed in packets/slot. Given that $R_{[\bar{u}]}^{(P)}$ may be not integer, we assume that the channel capacity can be either $D_{\bar{u}} \equiv\left\lfloor R_{[\bar{u}]}^{(P)}\right\rfloor$ with probability $p_{\bar{u}}=1-\left(R_{[\bar{u}]}^{(P)}-D_{\bar{u}}\right)$, or $D_{\bar{u}} \equiv\left\lfloor R_{[\bar{u}]}^{(P)}\right\rfloor+1 \quad$ with probability $1-p_{\bar{u}}=R_{[\bar{u}]}^{(P)}-D_{\bar{u}}$. Therefore, the emission probability matrix of the SBBP modeling the channel can be calculated as follows:

$$
B_{[\bar{u}, d]}^{(\tilde{N})}= \begin{cases}p_{\bar{u}} & \text { if } d=D_{\bar{u}} \\ p_{\bar{u}+1} & \text { if } d=D_{\bar{u}}+1 \\ 0 & \text { otherwise }\end{cases}
$$

The maximum number of packets that can be transmitted in one slot is $r_{M A X}^{(\widetilde{N})}=\max _{i}\left\{D_{i}+1\right\}$.

\subsection{Rate-controlled video source model}

As shown in Fig. 1, the Video Source System Model is constituted by the Adaptive-Rate Source and the transmission buffer. The adaptive-rate source pursues a given target by implementing a feedback law, $q=\phi\left(s_{Q}, a, j\right)$, where $s_{Q}$ is the transmission buffer state, $a$ and $j$ are the activity level and the encoding mode ( $\mathrm{I}, \mathrm{P}$ or $\mathrm{B}$ ) of the frame to be encoded. Thanks to this law, the rate controller calculates the value $q$ of the QSP to be used by the MPEG encoder for each frame.

In order to model the RCMV source as a whole, indicated here as $\Sigma$, we define the state of the whole system as $S^{(\Sigma)}(n)=\left(S^{(Q)}(n), S^{(\tilde{N})}(n), S^{(\tilde{Y})}(n)\right)$, where:

- $S^{(Q)}(n)$ is the transmission buffer queue state in the $n$-th slot, i.e., the number of packets in the queue and in the service facility at the observation instant;

- $S^{(\tilde{N})}(n)$ is the state of the underlying Markov chain of the channel SBBP $\tilde{N}(n)$, that is, $S^{(\widetilde{N})}(n)=U(n)$;

- $S^{(\widetilde{Y})}(n)$ is the state of the underlying Markov chain of $\widetilde{Y}(n)$, coinciding with that of $\widetilde{Y}_{q}(n)$, for any $q \in[1,31]$.

The transmission buffer state in the slot $n+1$ can be obtained through the Lindley equation:

$$
s_{Q}^{\prime \prime}=\max \left\{\min \left\{s_{Q}^{\prime}+r, K\right\}-d, 0\right\}
$$

where $s_{Q}^{\prime}$ is the transmission buffer state in the generic slot $n$, while $r$ and $d$ are the server capacity and the number of arrivals at the slot $n+1$.

The channel SBBP $\tilde{N}(n)$, modeled so far, can be equivalently characterized through the set of transition probability matrices, $M^{(\widetilde{N})}(d)$, which are transition probability matrices including the probability that the server capacity is $d$ (in [packets/slot]). Their generic element is defined as follows:

$$
\begin{aligned}
& \left|M^{(\tilde{N})}(d)\right|_{\left[s_{\tilde{N},}^{(1)}, s_{\tilde{N}}^{(2)}\right]} \equiv \\
& \equiv \operatorname{Prob}\left\{\begin{array}{l}
\tilde{N}(n+1)=d, \\
S^{(\tilde{N})}(n+1)=s_{\tilde{N}}^{(2)}
\end{array} \mid S^{(\tilde{N})}(n)=s_{\tilde{N}}^{(1)}\right\}=
\end{aligned}
$$

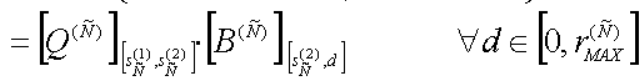

The RCMV source emission process is modeled by an SBBP whose emission probability matrix de- 
pends on the transmission buffer state. In order to model this process, we use the SBBP model of the non-controlled MPEG video source described in Section $2, \widetilde{Y}_{q}(n)$, for each $q \in[1,31]$. So we have a parameter set $\left(Q^{(\tilde{Y})}, B^{\left(\tilde{Y}_{1}\right)}, B^{\left(\tilde{Y}_{2}\right)}, \ldots, B^{\left(\widetilde{Y}_{31}\right)}\right)$, which represents an SBBP whose transition matrix is $Q^{\left(\hat{Y}^{\tilde{Y}}\right)}$, and whose emission process is characterized by a set of emission matrices, $\left\{B^{\left(\tilde{Y}_{q}\right)}\right\}_{q=1 . \ldots 31}$. At each time slot, the emission of the MPEG video source is therefore characterized by an emission probability matrix chosen according to the QSP value defined by the feedback law $q=\phi\left(s_{Q}, a, j\right)$. More concisely, as we did in (8) for the channel SBBP, we characterize the emission process of the RCMV source through the set of matrices $M_{s_{Q}^{(1)}, s_{\tilde{N}}^{(1)}}^{(\widetilde{Y})}(r), \forall r \in\left[0, r_{M A X}^{(\widetilde{Y})}\right]$, each matrix representing the transition probability matrix including the probability of $r$ packets being emitted when the buffer state is $s_{Q}^{(1)}$, and the channel state is $s_{\tilde{N}}^{(1)}$. So, the generic element of these matrices can be obtained from the above parameter set, $\left(Q^{(\widetilde{Y})}, B^{\left(\widetilde{Y_{1}}\right)}, B^{\left(\widetilde{Y}_{2}\right)}, \ldots, B^{\left(\tilde{Y}_{31}\right)}\right)$ as follows:

$$
\begin{aligned}
& {\left[M_{s_{Q}^{(1)}, s_{\tilde{N}}^{(1)}}^{(\tilde{y})}(r)\right]_{\left[\left(i^{(1)}, j^{(1)}\right) \cdot\left(\left(^{(2)}, j^{(2)}\right)\right]\right.}=}
\end{aligned}
$$

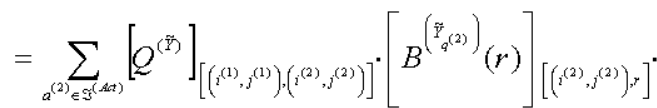

$$
\begin{aligned}
& \text { - } f_{A c t}\left(a^{\langle 2)} \mid i^{(2)}, j^{(2)}\right)
\end{aligned}
$$

where:

- $q^{(2)}=\phi\left(s_{Q}^{(1)}, a^{(2)}, j^{(2)}\right)$ is the QSP chosen when the frame to be encoded is the $j^{(2)}$-th in the GoP, the activity is $a^{(2)}$, and the transmission buffer state before encoding this frame is $s_{Q}^{(1)}$;

- $f_{A c t}\left(a^{(2)} \mid i^{(2)}, j^{(2)}\right)$ is the probability that the generic frame $j^{(2)}$ in the GoP has an activity $a^{(2)}$ when its activity level is $i^{(2)}$. This function, as demonstrated in $[17,18,24]$, is a Gamma probability density function, whose mean value and variance characterize the video trace;

- $\mathfrak{I}^{(A c t)}$ is the set of all the possible activities.

Finally, we can model the RCMV source as a whole. If we indicate two generic states of the system as $s_{\Sigma}^{(1)}=\left(s_{Q}^{(1)}, s_{\tilde{N}}^{(1)}, s_{\tilde{Y}}^{(1)}\right)$ and $s_{\Sigma}^{(2)}=\left(s_{Q}^{(2)}, s_{\tilde{N}}^{(2)}, s_{\tilde{Y}}^{(2)}\right)$, the generic element of the transition matrix of the video transmission system as a whole can be calculated, as follows:

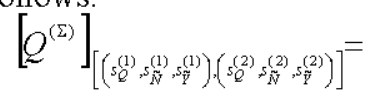

$$
\begin{aligned}
= & \sum_{d=0}^{d_{M A N A}^{(\tilde{N})}} \sum_{r=0}^{r_{\text {MAAX }}^{(\tilde{N})}}\left[M^{(\widetilde{N})}(d)\right]_{\left[s_{\tilde{N}}^{(1)}, s_{\tilde{N}}^{(2)}\right]} \cdot\left[M_{s_{Q}^{(1)}, s_{\tilde{N}}^{(1)}}^{(\widetilde{Y})}(r)\right]_{\left[s_{\tilde{Y}}^{(1)}, s_{\tilde{Y}}^{(2)}\right]} \cdot \\
& \cdot \psi\left(s_{Q}^{(1)}, s_{Q}^{(2)}, K, r, d\right)
\end{aligned}
$$

where $\psi\left(s_{Q}^{(1)}, s_{Q}^{(2)}, K, r, d\right)$ is a Boolean condition for the queue state behavior and is defined as follows: $\psi\left(s_{Q}^{(1)}, s_{Q}^{(2)}, K, r, d\right)= \begin{cases}1 & \text { if } \max \left\{\min \left\{s_{Q}^{(1)}+r, K\right\}\right. \\ 0 & \text { otherwise }\end{cases}$

Once the matrix $Q^{(\Sigma)}$ is known, we can calculate the steady-state probability array of the system $\Sigma$ as the solution of the following linear system:

$$
\underline{\pi}^{(\Sigma)} \cdot Q^{(\Sigma)}=\underline{\pi}^{(\Sigma)} \quad \text { with } \quad \underline{\pi}^{(\Sigma)} \cdot \underline{1}=1
$$

where 1 is a column array all of whose elements are equal to 1 , and $\underline{\pi}^{(\Sigma)}=\left[\underline{\pi}_{[0]}^{(\Sigma)}, \underline{\pi}_{[1]}^{(\Sigma)}, \ldots, \underline{\pi}_{[K-D]}^{(\Sigma)}\right]$ is the steady-state probability array, whose generic element, $\underline{\pi}_{\left[s_{Q}\right]}^{(\Sigma)}$, is the array containing the steady-state probabilities of the source when the virtual buffer is in the state $s_{Q}$. It may be difficult to solve (11) directly since the number of states grows explosively as the transmission buffer size range increases. In this paper we resort to feedback-type first order Markov fluid queueing models to approximate the behavior of adaptive MPEG servers [6]. In an ordinary Markov fluid queue the net drift of the queue changes with respect to a continuous-time Markov chain. In a feedback-type fluid queue, the net input rate not only depends on the state of the Markov chain but also the instantaneous queue occupancy. For the purposes of modeling the RCMV source, we construct a continuous-time Markov fluid queue with the same state-space as above. The drifts in each state are calculated as the mean bit rates for each chosen quantization parameter $q \in[1,31]$. Depending on the adaptive policy used, regions of queue occupancy where the QSP stays constant for each state will be determined. This model, in principle, can analytically be solved as in [6]. However, the spectral decomposition approach used in [6] is prone to numerical errors especially for large-scale problems like the one studied in this paper. Therefore, we propose to use the ordered Schur decomposition [7] and the spectral divide and conquer problem of [8] as a means of solving the feedback fluid queue.

\section{CASE STUDY}

Let us now apply the model proposed in the paper to evaluate performance achieved by a mobile video user when it is in a UMTS cell. The case study considered here regards a UMTS cell which accepts at most twenty mobile video users, in order to guarantee them the required quality. We assume a system bandwidth of $W=3.84 \mathrm{Mcps}$, a user birth death rate 


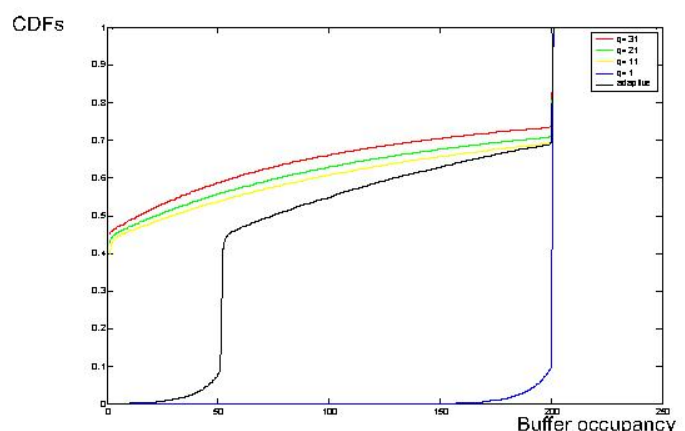

Figure 2. Cumulative distribution function of the buffer occupancy for four static policies and one adaptive policy

of $0.01667 \mathrm{~s}^{-1}$, a threshold of $\eta_{u L}^{T h}=0.75$, and a value of bit energy over noise ratio for each user given by $\left(E_{b} / N_{0}\right)^{M N}=2 \mathrm{~dB}$, relating to a fast fading channel with users moving at $3 \mathrm{~km} / \mathrm{h}$. Table 1 shows the maximum bit rate that can be achieved by each user vs. the number of users active in the cell, calculated according to (5)

The SBBP model of the video source was calculated by one hour of MPEG video sequences of the movie "The Silence of the Lambs." To encode this movie, we used a frame rate of $24 \mathrm{frames} / \mathrm{s}$, a frame size of $M=180$ macroblocks. The GoP structure $I B B P B B$ was used. The Transmission Buffer size was assumed of 200 packets, with a packet size of 576 bytes. We study an adaptive MPEG server system sharing a UMTS uplink with 19 other video sources using the feedback-type Markov fluid queueing model. In this example, we study four static policies for each of which the quantization scale parameter QSP is fixed for all buffer occupancies. We use the QSP settings 1, 11, 21 and 31 for each static server policy. In the adaptive case, there are four regions of the buffer occupancy; the QSP is chosen to be 1, 11, 21 and 31 for all types of frames when the number of packets in the buffer is within the intervals [0 50], (50 100], (100 150], (151, 200], respectively, where 200 is the maximum number of packets that the buffer can hold. The cumulative distribution function of the queue occupancy is given in Fig. 2. For $\mathrm{QSP}=1$, the net input rate to the queue is excessively large leading to large loss rates irrespective of the frame type. For the other studied QSP parameters the loss rate is significantly reduced but at the expense of both reduced encoding video quality and increased probabilities of empty buffers which account for wasted bandwidth. On the other hand, the adaptive policy tries to steer the buffer occupancy from the two boundaries although there still is accumulation at the right boundary that accounts for random losses of all types of frames. In the future, we plan on exploring a wide variety of adaptive policies and their impact on overall PSNR and visual quality so as to be able to provide guidelines for operating adaptive MPEG systems for UMTS environments.

\begin{tabular}{|c|c|}
\hline Users & Rate per user \\
\hline 3 & $425,21 \mathrm{~kb} / \mathrm{s}$ \\
\hline 4 & $294,37 \mathrm{~kb} / \mathrm{s}$ \\
\hline 5 & $225,11 \mathrm{~kb} / \mathrm{s}$ \\
\hline 6 & $182,23 \mathrm{~kb} / \mathrm{s}$ \\
\hline 7 & $153,07 \mathrm{~kb} / \mathrm{s}$ \\
\hline 8 & $131,96 \mathrm{~kb} / \mathrm{s}$ \\
\hline 9 & $115,97 \mathrm{~kb} / \mathrm{s}$ \\
\hline 10 & $103,43 \mathrm{~kb} / \mathrm{s}$ \\
\hline 11 & $93,34 \mathrm{~kb} / \mathrm{s}$ \\
\hline
\end{tabular}

\begin{tabular}{|c|c|}
\hline Users & Rate per user \\
\hline 12 & $85,04 \mathrm{~kb} / \mathrm{s}$ \\
\hline 13 & $78,10 \mathrm{~kb} / \mathrm{s}$ \\
\hline 14 & $72,20 \mathrm{~kb} / \mathrm{s}$ \\
\hline 15 & $67,14 \mathrm{~kb} / \mathrm{s}$ \\
\hline 16 & $62,74 \mathrm{~kb} / \mathrm{s}$ \\
\hline 17 & $58,87 \mathrm{~kb} / \mathrm{s}$ \\
\hline 18 & $55,46 \mathrm{~kb} / \mathrm{s}$ \\
\hline 19 & $52,42 \mathrm{~kb} / \mathrm{s}$ \\
\hline 20 & $49,70 \mathrm{~kb} / \mathrm{s}$ \\
\hline
\end{tabular}

Table 1. Bit rates vs. the number of active users

\section{CONCLUSIONS}

The paper proposes a new Markov-based model of real-time video sources in a UMTS environment, to examine effects of the cell conditions on the performance of video communications. The model is applied to compare different feedback laws implemented by the rate controller to control the output video flow. However the model can be applied to the design of both the source encoding parameters, such as the feedback law, and some network parameters, such as the maximum number of users in a cell.

\section{REFERENCES}

[1] L. J. Lin et al., "Bit-rate control using piecewise approximated rate-distortion characteristics," IEEE Trans. on Circuits and Syst. for Video Tech., 38-1, 1990.

[2] W. Ding and B. Liu, "Rate Control of MPEG Video Coding and Recording by RateQuantization Modeling," IEEE Transactions on Circuits and Systems for Video Technology, Vol. 6, No. 1, February 1996.

[3] A. Lombardo, G. Schembra, "Performance evaluation of an Adaptive-Rate MPEG encoder matching IntServ Traffic Constraints," IEEE Transactions on Networking, vol. 11, no. 1, pp. 47-65, February 2003.

[4] A. Lombardo, G. Morabito, G. Schembra, "An Accurate and Treatable Markov Model of MPEG-Video Traffic", IEEE Proc. Infocom '98, San Francisco, USA, 1998.

[5] Harri Holma and Antti Toskala, "WCDMA for UMTS", John Wiley \& Sons, 3rd Edition, 2004.

[6] A. I. Elwalid and D. Mitra, "Fluid Models for the Analysis and Design of Statistical Multiplexing with Loss Priorities on Multiple Classes of Bursty Traffic", Infocom '92, 1992, pp. 415-425.

[7] N. Akar, N. C. Oguz, and K. Sohraby, A Novel Computational Method for Solving Finite QBD Processes, Stochastic Models, vol. 16, no.2, 2000.

[8] N. Akar and K. Sohraby, "Infinite- and FiniteBuffer Markov Fluid Queues: A Unified Analysis", J. Appl. Prob. Vol. 41, no. 2. 